\title{
Triphenylphosphine promoted addition of acetylenic esters to benzofuran-2,3-dione: one-pot synthesis of Novel $\gamma$ - Spirolactones
}

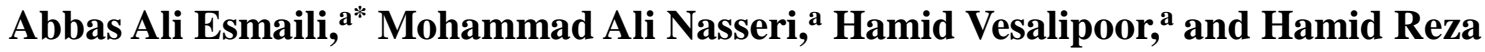 \\ Bijanzadeh $^{\text {b }}$ \\ ${ }^{a}$ Department of Chemistry, University of Birjand, P. O. Box 97175/615, Birjand, Iran \\ ${ }^{b}$ Department of Chemistry, Tarbiat Modares University, P.O. Box 14115-175, Tehran, Iran \\ E-mail: $\underline{\text { aa_esmaeili@yahoo.com }}$
}

\begin{abstract}
The reaction of the zwitterionic intermediates generated from dialkyl acetylene dicarboxylates and triphenylphosphine with benzofuran-2,3-diones provides a one-pot route to new highly functionalized $\gamma$-spirolactones in good yield.
\end{abstract}

Keywords: $\gamma$-Spirolactones, benzofuran-2,3-diones, acetylenic esters, triphenylphosphine

\section{Introduction}

The 2,5-dihydrofuran-2-one moiety is an integral part of many natural and unnatural products. Recently, it has been reported that derivatives of 2,5-dihydrofuran-2-one show biological activity. ${ }^{1-4}$ For example 2,5-dihydrofuran-2-one derivatives $\mathbf{1}$ and $\mathbf{2}$ have antimicrobial activities. ${ }^{5}$<smiles>[R]C(=O)C1=C(O)C(=O)OC1Br</smiles>

1<smiles>[R]C(=O)C1=C(O)C(=O)OC12C(=O)c1ccccc1C2=O</smiles>

2

Recently Chatani and co-workers reported that the reaction of benzofuran-2,3-dione derivatives with $\mathrm{CO}$ and alkynes via a ruthenium-catalyzed carbonylative [2+2+1] cycloaddition provides a general route to $\gamma$-spiro-lactones. ${ }^{6}$ 
Multi-Component reactions (MCRs), in which several different starting materials can be combined in one reaction to give a highly complex product, are finding increasing use in modern organic chemistry. ${ }^{7-9}$ They offer significant advantages over conventional linear syntheses by reducing time and by saving money, energy, and raw materials, thus providing both economic and environmental benefits. At the same time, diversity can be achieved from building up libraries by simply varying each component. ${ }^{10-15}$

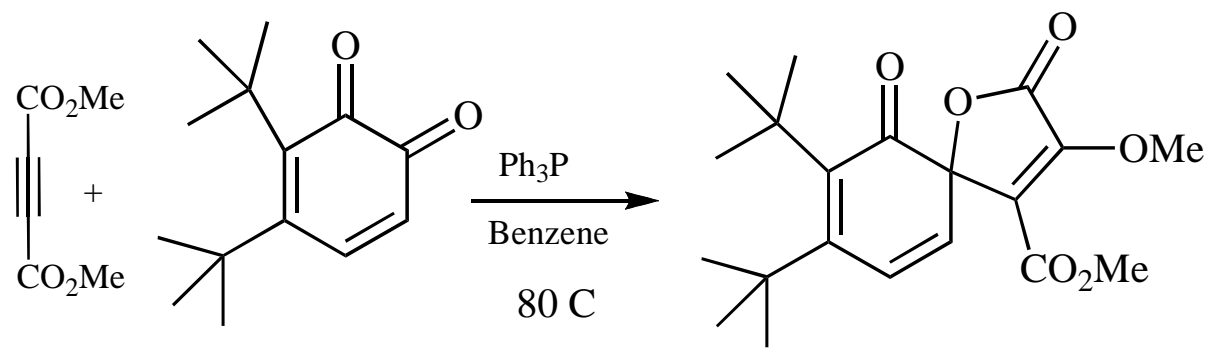

\section{Scheme 1}

The early work of Nair and coworkers showed that the zwitterionic intermediates formed between dialkyl acetylenedicarboxylates and $\mathrm{Ph}_{3} \mathrm{P}$ react with activated dicarbonyl compounds to afford highly functionalized $\gamma$-butyrolactones (Scheme 1). ${ }^{16-18}$ We have also demonstrated that the activated carbonyl of phenyl glyoxalate and isatin derivatives can take part in such reactions successfully, thus constituting a ready synthesis of heterocyclic compounds. ${ }^{19-20}$

We now wish to report a simple one-pot three-component reaction between dialkyl acetylenedicarboxylates, $\mathrm{Ph}_{3} \mathrm{P}$, and benzofuran-2,3-dione derivatives leading to methyl 4'methoxy-spiro[benzofuran-3,2'-furan]-2,5'-dione-3'-carboxylate derivatives as $\gamma$ spirolactone systems (Scheme 2 and Table 1).

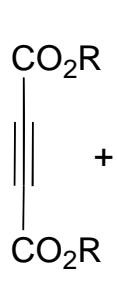

3<smiles>[R]c1cc([R])c2c(c1[R])OC(=O)C2=O</smiles>

4<smiles>[R]OC(=O)C1=C([R])C(=O)O[C@]12C(=O)Oc1c([R7])c([R])cc([R])c12</smiles>

5

Scheme 2 
Table 1. The reaction of benzofuran-2,3-dione $\mathbf{4}$ with dialkyl acetylenedicarboxylate $\mathbf{3}$ in the presence of $\mathrm{Ph}_{3} \mathrm{P}$

\begin{tabular}{cccccc}
\hline Product & $\mathrm{R}^{1}$ & $\mathrm{R}^{2}$ & $\mathrm{R}^{3}$ & $\mathrm{R}$ & \% Yield of $\mathbf{5}$ \\
\hline $\mathbf{5 a}$ & $\mathrm{Me}$ & $\mathrm{H}$ & $\mathrm{Me}$ & $\mathrm{Me}$ & 50 \\
$\mathbf{5 b}$ & $\mathrm{Me}$ & $\mathrm{H}$ & $\mathrm{Me}$ & $\mathrm{Et}$ & 55 \\
$\mathbf{5 c}$ & $\mathrm{Me}$ & $\mathrm{Me}$ & $\mathrm{H}$ & $\mathrm{Me}$ & 60 \\
$\mathbf{5 d}$ & $\mathrm{Me}$ & $\mathrm{Me}$ & $\mathrm{H}$ & $\mathrm{Et}$ & 75 \\
$\mathbf{5 e}$ & $\mathrm{Me}$ & $\mathrm{Me}$ & $\mathrm{H}$ & $\mathrm{CHMe}_{2}$ & 85 \\
$\mathbf{5 f}$ & $\mathrm{Me}$ & $\mathrm{Me}$ & $\mathrm{Me}$ & $\mathrm{Et}$ & 44 \\
\hline
\end{tabular}

\section{Results and Discussion}

In this type of two-component reaction catalyzed by nucleophiles, triphenylphosphine $\left(\mathrm{Ph}_{3} \mathrm{P}\right)$ has been the most often studied nucleophilic species. As early as 1961, Tebby observed that the addition of $\mathrm{Ph}_{3} \mathrm{P}$ to various activated alkynes like DMAD, dicyanoacetylene, and dibenzoylacetylene generates zwitterionic intermediates. ${ }^{21,22}$

The reactions were initiated by the addition of a solution of dimethyl acetylenedicarboxylate (3) (1.1 equiv in $3 \mathrm{ml} \mathrm{CH}_{2} \mathrm{Cl}_{2}$ ) to a solution of benzofuran-2,3-dione derivatives 4 (1 equiv.) and $\mathrm{Ph}_{3} \mathrm{P}$ in $\mathrm{CH}_{2} \mathrm{Cl}_{2}(5 \mathrm{ml})$ at $-10{ }^{\circ} \mathrm{C}$. The mixture was stirred for 1 h. Distillation of the solvent in vacuum followed by addition of cold $\mathrm{Et}_{2} \mathrm{O}$ or $\mathrm{EtOH}$ led to a crystalline product 5 in $45-85 \%$ yield (Scheme 2).

The products 5a-f were characterized on the basis of their analytical and spectroscopic data. For example, the IR spectrum of $\mathbf{5 a}$ displayed characteristic ester $\mathrm{C}=\mathrm{O}$ absorptions at 1760, 1750 and $1720 \mathrm{~cm}^{-1}$ respectively. In the ${ }^{1} \mathrm{H}$ NMR spectrum, the two aromatic Me groups appeared as sharp singlets at $\delta 2.37$ and $\delta 2.65$ and the $\mathrm{MeO}$ groups resonated at $\delta$ 3.89 and $\delta$ 4.27. The ${ }^{13} \mathrm{C}$ NMR spectrum of 5a displayed sixteen distinct signals in agreement with the proposed structure. Partial assignment of these resonances is given in the Experimental Section. The characteristic signal of the spiro carbon atom was discernable at 89.36 . Finally, the $\gamma$-spirolactone formation can be rationalized as shown in Scheme 3. 


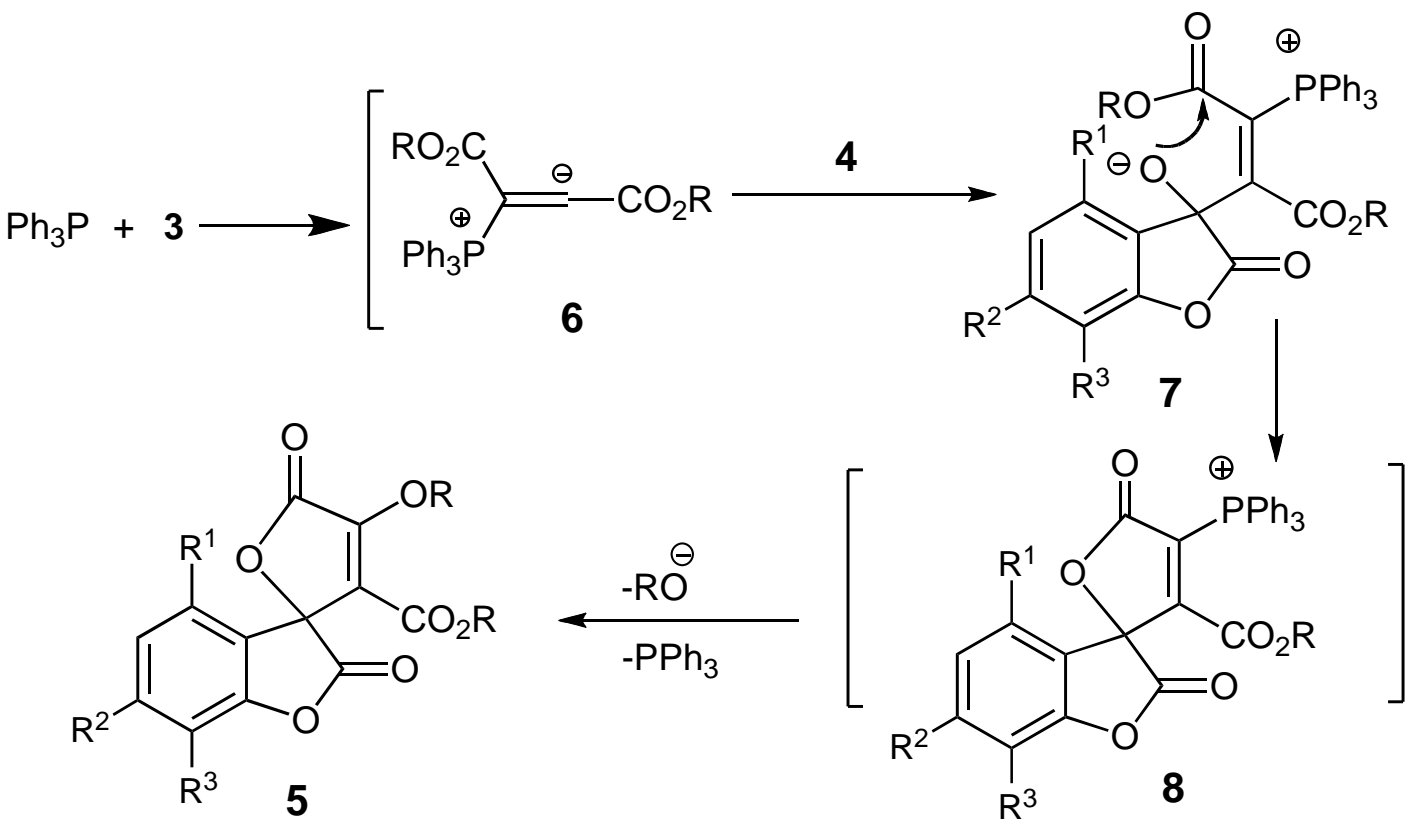

\section{Scheme 3}

\section{Conclusions}

In summary, we have succeeded in synthesizing $\gamma$-spirolactones of potential synthetic interest via a one-pot reaction between dialkyl acetylenedicarboxylates and benzofuran-2,3dione in the presence of triphenylphosphine. High yields of the products, relatively short reaction times, and use of simple starting materials are the main advantages of this method. The reactions were performed under neutral and mild conditions, and the starting materials and reagents can be reacted without any activation or modification.

\section{Experimental Section}

Melting points were measured on an Electrothermal 9100 apparatus and are uncorrected. Elemental analyses for $\mathrm{C}, \mathrm{H}$, and $\mathrm{N}$ were performed using a Heraeus CHN-O-Rapid analyzer. IR spectra were measured on a Perkin-Elmer 783 Infrared spectrophotometer. ${ }^{1} \mathrm{H}$ and ${ }^{13} \mathrm{C}$ NMR spectra were measured with a BRUKER DRX-500 AVANCE spectrometer at 500 and $125.77 \mathrm{MHz}$. Mass spectra were recorded on a SHIMADZU GCMS-QP5050 mass spectrometer operating at an ionization potential of $70 \mathrm{eV}$. Triphenylphosphine, and acetylenic esters 3 were obtained from Fluka (Buchs, Switzerland) and were used without purification. 
General procedure for the preparation of alkyl-4'-alkoxy-spiro[benzofuran-3,2'furan]-2,5'dione-3'-carboxylate derivatives 5

To a stirred solution of 3,5-dimethyl benzofuran-2,3-dione derivatives 4 (0.220 g, 1.0 mmol) and triphenylphosphine $(0.262 \mathrm{~g}, 1 \mathrm{mmol})$ in dry $\mathrm{CH}_{2} \mathrm{Cl}_{2}(5 \mathrm{ml})$ was added dropwise a solution of dimethyl acetylenedicarboxylate $3(0.141 \mathrm{~g}, 1 \mathrm{mmol})$ in dry $\mathrm{CH}_{2} \mathrm{Cl}_{2}(2 \mathrm{ml})$ at $10{ }^{\circ} \mathrm{C}$ over $10 \mathrm{~min}$ via a syringe, and the reaction mixture was then allowed to warm up to room temperature and stirred for about $20 \mathrm{~min}$. On completion of the reaction, solvent was removed under vacuum and to the residues, cold diethyl ether $(5 \mathrm{ml})$ for $\mathbf{5 a}$ and cold ethanol $(5 \mathrm{ml})$ for $\mathbf{5 b - 5 f}$, were added to produce pure solid products. Then, the resulting products were filtered off to afford the spirolactone 5 .

Methyl-4,7-dimethyl-4'-methoxy-spiro[benzofuran-3,2'-furan]-2,5'dione-3'carboxylate (5a). Yield: $0.159 \mathrm{~g}(50 \%), \mathrm{mp} 202-204{ }^{\circ} \mathrm{C} ;{ }^{1} \mathrm{H} \mathrm{NMR}\left(300 \mathrm{MHz}, \mathrm{CDCl}_{3}\right): \delta=$ 2.37, $2.65\left(6 \mathrm{H}, 2 \mathrm{~s}, 2 \mathrm{CH}_{3}\right), 3.89,4.27(6 \mathrm{H}, 2 \mathrm{~s}, 2 \mathrm{OMe}), 6.93\left(1 \mathrm{H}, \mathrm{d},{ }^{3} J_{H H}=7.6 \mathrm{~Hz}, \mathrm{H} 5\right)$, $7.13\left(1 \mathrm{H}, \mathrm{d},{ }^{3} J_{H H}=7.6 \mathrm{~Hz}, \mathrm{H} 6\right),{ }^{13} \mathrm{C} \mathrm{NMR}\left(75.46 \mathrm{MHz}, \mathrm{CDCl}_{3}\right) ; \delta=15.7$ and $20.6\left(2 \mathrm{CH}_{3}\right)$, 51.8 (OMe), 59.2 (OMe), 89.4 (C Spiro), 108.9 (C3a), 110.2 (C7), 123.8 (C5), 125.9 (C6), 130.1 (C4), 131.0 (C7a), 150.3 (C4'), 151.0 (C3'), 155.2, 161.7, 163.1 (3 C=O); MS, m/z (\%): $302\left(\mathrm{M}^{+}-1-\mathrm{Me}, 100\right), 287\left(\mathrm{M}^{+}-\mathrm{OMe}, 97\right), 259\left(\mathrm{M}^{+}-\mathrm{CO}_{2} \mathrm{Me}, 13\right), 227\left(\mathrm{M}^{+}+1-\mathrm{CO}_{2} \mathrm{Me}-\right.$ OMe, 33), $199\left(\mathrm{M}^{+}+1-2 \mathrm{CO}_{2} \mathrm{Me}, 27\right), 159\left(\mathrm{C}_{10} \mathrm{H}_{10} \mathrm{O}_{2}{ }^{+}, 4\right), 172\left(\mathrm{M}^{+}-\mathrm{OMe}-\mathrm{CO}_{2} \mathrm{Me}-2 \mathrm{Me}-\mathrm{CO}\right.$, 12), $91\left(\mathrm{C}_{7} \mathrm{H}_{8}{ }^{+}, 18\right), 59\left(\mathrm{CO}_{2} \mathrm{Me}^{+}, 11\right)$; IR (KBr) $\left(v_{\max }, \mathrm{cm}^{-1}\right): 3010\left(\mathrm{C}-\mathrm{H}_{\mathrm{St}}\right), 1760,1750$, $1720(3 \mathrm{C}=\mathrm{O}), 1580,1480(\mathrm{C}=\mathrm{C})\left(\right.$ arom); Anal. calc. for $\mathrm{C}_{16} \mathrm{H}_{14} \mathrm{O}_{7}$ (318.28): C 60.38, $\mathrm{H}$ $4.43 \%$ found: C $60.0, \mathrm{H} 4.3 \%$.

Ethyl-4,7-dimethyl-4' -ethoxy-spiro[benzofuran-3,2'-furan]-2,5'dione-3'-carboxylate (5b). Yield: $0.191 \mathrm{~g}(55 \%), \mathrm{mp} 119-120{ }^{\circ} \mathrm{C} ;{ }^{1} \mathrm{H} \mathrm{NMR}\left(500 \mathrm{MHz}, \mathrm{CDCl}_{3}\right): \delta=1.37(3 \mathrm{H}, \mathrm{t}$, ${ }^{3} J_{H H}=7.1 \mathrm{~Hz}, \mathrm{CH}_{3}$, of ester), $1.52\left(3 \mathrm{H}, \mathrm{t},{ }^{3} \mathrm{~J}_{\mathrm{HH}}=7.1 \mathrm{~Hz}, \mathrm{CH}_{3}\right.$, of ether $), 2.37,2.62(6 \mathrm{H}, 2 \mathrm{~s}$, $2 \mathrm{Me}), 4.34\left(2 \mathrm{H}, \mathrm{q},{ }^{3} J_{H H}=7.1 \mathrm{~Hz}, \mathrm{CH}_{2}\right.$, of ester), $4.57\left(2 \mathrm{H}, \mathrm{q},{ }^{3} J_{H H}=7.1 \mathrm{~Hz}, \mathrm{CH}_{2}\right.$, of ether), $6.92\left(1 \mathrm{H}, \mathrm{d},{ }^{3} J_{H H}=7.6 \mathrm{~Hz}, \mathrm{H} 5\right), 7.12\left(1 \mathrm{H}, \mathrm{d},{ }^{3} J_{H H}=7.6 \mathrm{~Hz}, \mathrm{H} 6\right) ;{ }^{13} \mathrm{C} \mathrm{NMR}(125.75$ $\left.\mathrm{MHz}, \mathrm{CDCl}_{3}\right) ; \delta=14.2$ and $15.0\left(2 \mathrm{CH}_{3}\right), 15.8$ and $20.7\left(2 \mathrm{CH}_{3}\right), 60.8,69.6\left(2 \mathrm{OCH}_{2}\right)$, 91.9 (CSpiro), 109.0 (C3a) 110.5 (C6) 124.0 (C7), 125.9 (C4), 130.2 (C5), 131.0 (C7a), 150.5 (C4'), 151.3(C3'), 155.4, 161.5, 162.7 (3 C=O); MS, m/z (\%): $330\left(\mathrm{M}^{+}-1-\mathrm{Me}, 20\right)$, $301\left(\mathrm{M}^{+}-\mathrm{OCH}_{2} \mathrm{CH}_{3}, 9\right), 256\left(\mathrm{M}^{+}-1-\mathrm{OCH}_{2} \mathrm{CH}_{3}-\mathrm{CH}_{2} \mathrm{CH}_{3}-\mathrm{CH}_{3}, 100\right), 201\left(\mathrm{M}^{+}-\mathrm{OCH}_{2} \mathrm{CH}_{3}^{-}\right.$ $\left.\mathrm{CH}_{2} \mathrm{CH}_{3}-\mathrm{CH}_{3}-\mathrm{C}-\mathrm{CO}_{2}, 11\right), 91\left(\mathrm{C}_{7} \mathrm{H}_{8}{ }^{+}, 15\right)$; IR $(\mathrm{KBr})\left(v_{\max }, \mathrm{cm}^{-1}\right): 3008\left(\mathrm{C}-\mathrm{H}_{\mathrm{St}}\right), 1750,1745$, $1700(3 \mathrm{C}=\mathrm{O}), 1550,1435(\mathrm{C}=\mathrm{C})$ (arom); Anal. calc. for $\mathrm{C}_{18} \mathrm{H}_{18} \mathrm{O}_{7}$ (346.34): $\mathrm{C}$ 62.42, $\mathrm{H}$ $5.24 \%$ found: C 62.6, H $5.1 \%$.

Methyl-4,6-dimethyl-4'-methoxy-spiro[benzofuran-3,2'-furan]-2,5'dione-3'carboxylate (5c). Yied: $0.191 \mathrm{~g}(60 \%), \mathrm{mp} 198-199{ }^{\circ} \mathrm{C} ;{ }^{1} \mathrm{H} \mathrm{NMR}\left(500 \mathrm{MHz}, \mathrm{CDCl}_{3}\right): \delta=$ 2.27, 2.52 (6H, 2 s, $\left.2 \mathrm{CH}_{3}\right), 3.81,4.19(6 \mathrm{H}, 2 \mathrm{~s}, 2 \mathrm{OMe}), 6.75(1 \mathrm{H}, \mathrm{s}, \mathrm{H} 5), 6.79(1 \mathrm{H}, \mathrm{s}, \mathrm{H} 7)$, ${ }^{13} \mathrm{C} \mathrm{NMR}\left(125.75 \mathrm{MHz}, \mathrm{CDCl}_{3}\right) ; \delta=20.6$ and $21.4\left(2 \mathrm{CH}_{3}\right), 51.8(\mathrm{OMe}), 59.2(\mathrm{OMe}), 89.3$ (CSpiro), 107.9 (C3a), 108.1 (C6), 114.7 (C7), 127.7 (C5), 132.42 (C4), 140.7 (C7a), 150.2 (C4'), 152.9 (C3'), 155.4, 161.6, 162.9 (3 C=O); MS, m/z (\%): $302\left(\mathrm{M}^{+}-1-\mathrm{Me}, 73\right), 287$ $\left(\mathrm{M}^{+}-\mathrm{OMe}, 100\right), 227\left(\mathrm{M}^{+}+1-\mathrm{CO}_{2} \mathrm{Me}-\mathrm{OMe}, 48\right), 199\left(\mathrm{M}^{+}+1-2 \mathrm{CO}_{2} \mathrm{Me}, 36\right), 172\left(\mathrm{M}^{+}-\mathrm{OMe}-\right.$ 
$\left.\mathrm{CO}_{2} \mathrm{Me}-2 \mathrm{Me}-\mathrm{CO}, 13\right), 91\left(\mathrm{C}_{7} \mathrm{H}_{8}{ }^{+}, 54\right), 59\left(\mathrm{CO}_{2} \mathrm{Me}^{+}, 38\right)$; IR (KBr) $\left(v_{\max }, \mathrm{cm}^{-1}\right): 3010(\mathrm{C}-$ $\left.\mathrm{H}_{\mathrm{St}}\right), 1748,1735,1710(3 \mathrm{C}=\mathrm{O}), 1550,1430(\mathrm{C}=\mathrm{C})$ (arom); Anal. calc. for $\mathrm{C}_{16} \mathrm{H}_{14} \mathrm{O}_{7}$ (318.28): C 60.38, H $4.43 \%$ found: C 60.5, H $4.5 \%$.

Ethyl-4,6-dimethyl-4'-ethoxy-spiro[benzofuran-3,2'-furan]-2,5'dione-3'-carboxylate (5d). Yield: $0.259 \mathrm{~g}(75 \%), \mathrm{mp} 113-114{ }^{\circ} \mathrm{C} ;{ }^{1} \mathrm{H}$ NMR $\left(500 \mathrm{MHz}, \mathrm{CDCl}_{3}\right): \delta=1.36(3 \mathrm{H}, \mathrm{t}$, ${ }^{3} J_{H H}=7.1 \mathrm{~Hz}, \mathrm{CH}_{3}$, of ester), $1.51\left(3 \mathrm{H}, \mathrm{t},{ }^{3} J_{H H}=7.1 \mathrm{~Hz}, \mathrm{CH}_{3}\right.$, of ether $), 2.35,2.64(6 \mathrm{H}, 2 \mathrm{~s}$, $2 \mathrm{Me}), 4.35\left(2 \mathrm{H}, \mathrm{q},{ }^{3} J_{H H}=7.1 \mathrm{~Hz}, \mathrm{CH}_{2}\right.$, of ester $), 4.56\left(2 \mathrm{H}, \mathrm{q},{ }^{3} J_{\mathrm{HH}}=7.0 \mathrm{~Hz}, \mathrm{CH}_{2}\right.$, of ether), $6.87(1 \mathrm{H}, \mathrm{s}, \mathrm{H} 5), 7.00(1 \mathrm{H}, \mathrm{s}, \mathrm{H} 7) ;{ }^{13} \mathrm{C} \mathrm{NMR}\left(125.75 \mathrm{MHz}, \mathrm{CDCl}_{3}\right) ; \delta=14.2$ and $14.9\left(2 \mathrm{CH}_{3}\right), 20.7$ and $21.5\left(2 \mathrm{CH}_{3}\right), 60.80,69.7\left(2 \mathrm{OCH}_{2}\right), 91.0\left(\mathrm{C}_{\text {Spiro }}\right), 108.3(\mathrm{C} 3 \mathrm{a})$, 108.4 (C6), 127.7 (C7), 131.8 (C4), 131.9 (C5), 132.1 (C7a), 150.3 (C3'), 150.6 (C4'), 155.7, 161.5, 169.6 (3 C=O); MS, $m / z(\%): 330\left(\mathrm{M}^{+}-1-\mathrm{Me}, 10\right), 301\left(\mathrm{M}^{+}-\mathrm{OCH}_{2} \mathrm{CH}_{3}, 6\right)$, $256\left(\mathrm{M}^{+}-1-\mathrm{OCH}_{2} \mathrm{CH}_{3}-\mathrm{CH}_{2} \mathrm{CH}_{3}-\mathrm{CH}_{3}, 52\right), 201\left(\mathrm{M}^{+}-\mathrm{OCH}_{2} \mathrm{CH}_{3}-\mathrm{CH}_{2} \mathrm{CH}_{3}-\mathrm{CH}_{3}-\mathrm{C}-\mathrm{CO}_{2}, 28\right)$, $91\left(\mathrm{C}_{7} \mathrm{H}_{8}{ }^{+}, 14\right), 59\left(\mathrm{CO}_{2} \mathrm{Me}^{+}, 6\right)$; IR (KBr) $\left(v_{\max }, \mathrm{cm}^{-1}\right): 3020$ (C-HST), 1760, 1745, 1705 (3 $\mathrm{C}=\mathrm{O}), 1595,1440(\mathrm{C}=\mathrm{C})\left(\right.$ arom); Anal. calc. for $\mathrm{C}_{18} \mathrm{H}_{18} \mathrm{O}_{7}$ (346.34): $\mathrm{C}$ 62.42, $\mathrm{H}$ 5.24\% found: C $62.2, \mathrm{H} 5.3 \%$.

Isopropyl-4,6-dimethyl-4' -isopropoxy-spiro[benzofuran-3,2'-furan]-2,5'dione-3'carboxylate (5e). Yield: $0.318 \mathrm{~g}(85 \%), \mathrm{mp} 196-198{ }^{\circ} \mathrm{C} ;{ }^{1} \mathrm{H}$ NMR $\left(500 \mathrm{MHz}, \mathrm{CDCl}_{3}\right): \delta=$ $1.31\left(6 \mathrm{H}, \mathrm{d},{ }^{3} J_{H H}=6.3 \mathrm{~Hz}, \mathrm{CH}_{3}\right.$, of ester), $1.43\left(6 \mathrm{H}, \mathrm{d},{ }^{3} J_{H H}=6.2 \mathrm{~Hz}, \mathrm{CH}_{3}\right.$, of ether) 2.27, $2.54\left(6 \mathrm{H}, 2 \mathrm{~s}, 2 \mathrm{CH}_{3}\right), 4.93\left(1 \mathrm{H}, \mathrm{m},{ }^{3} J_{H H}=6.2 \mathrm{~Hz} \mathrm{CH}\right.$ of ester $), 5.17\left(1 \mathrm{H}, \mathrm{m},{ }^{3} J_{H H}=6.3 \mathrm{~Hz}\right.$ $\mathrm{CH}$ of ether) $6.77(1 \mathrm{H}, \mathrm{s}, \mathrm{H} 5), 6.79(1 \mathrm{H}, \mathrm{s}, \mathrm{H} 7) ;{ }^{13} \mathrm{C} \mathrm{NMR}\left(125.75 \mathrm{MHz}, \mathrm{CDCl}_{3}\right) ; \delta=20.70$ and $21.5\left(2 \mathrm{CH}_{3}\right), 21.9$ and $22.4\left(\mathrm{CH}_{3}\right.$, of ester and ether), $68.3(\mathrm{OCH}$ ester $), 79.2(\mathrm{OCH}$ ether) 93.2 (C Spiro), 108.1 (C3a), 108.2 (C6), 114.8 (C7), 127.5 (C5), 132.4 (C4), 140.6 (C7a), 150.7 (C4'), 153.1 (C3'), 155.6, 160.9, 161.1 (3 C=O); MS, m/z (\%): 358 (M+1-Me, 4), $316\left(\mathrm{M}^{+}+1-\mathrm{O} i-\mathrm{Pr}, 8\right), 274\left(\mathrm{M}^{+}-\mathrm{C}-\mathrm{CO}_{2} i-\mathrm{Pr}, 10\right), 256\left(\mathrm{M}^{+}-1-\mathrm{CO}_{2} i-\mathrm{Pr}-2 \mathrm{Me}, 100\right), 201$ $\left(\mathrm{M}^{+}+1-\mathrm{CO}_{2} i-\mathrm{pPr}-\mathrm{O} i-\mathrm{Pr}-\mathrm{CO}, 5\right), 91\left(\mathrm{C}_{7} \mathrm{H}_{8}^{+}, 18.5\right)$; IR (KBr) ( $\left.v_{\max }, \mathrm{cm}-1\right): 3003\left(\mathrm{C}-\mathrm{H}_{\mathrm{St}}\right)$, 1755, 1745, $1710(3 \mathrm{C}=\mathrm{O}), 1590,1470(\mathrm{C}=\mathrm{C})$ (arom); Anal. calc. for $\mathrm{C}_{16} \mathrm{H}_{14} \mathrm{O}_{7}$ (374.39): C 64.16, H 5.92\% found: C $64.5, \mathrm{H} 6.0 \%$.

Ethyl-4,6,7-trimethyl-4'-ethoxy-spiro[benzofuran-3,2'-furan]-2,5'dione-3'-carboxylate (5f). Yield: $0.158 \mathrm{~g} \mathrm{(44 \% ),} \mathrm{mp} \mathrm{161-162}{ }^{\circ} \mathrm{C} ;{ }^{1} \mathrm{H}$ NMR (500 MHz, $\left.\mathrm{CDCl}_{3}\right): \delta=1.37(3 \mathrm{H}, \mathrm{t}$, ${ }^{3} J_{\mathrm{HH}}=7.1 \mathrm{~Hz}, \mathrm{CH}_{3}$, of ester), $1.51\left(3 \mathrm{H}, \mathrm{t},{ }^{3} J_{\mathrm{HH}}=7.1 \mathrm{~Hz}, \mathrm{CH}_{3}\right.$, of ether), 2.26, $2.26(6 \mathrm{H}, 2 \mathrm{~s}$, $\left.2 \mathrm{CH}_{3}\right), 2.56\left(3 \mathrm{H}, \mathrm{s}, \mathrm{CH}_{3}\right), 4.33\left(2 \mathrm{H}, \mathrm{q},{ }^{3} J_{\mathrm{HH}}=7.1 \mathrm{~Hz}, \mathrm{CH}_{2}\right.$, of ester $), 4.56\left(2 \mathrm{H}, \mathrm{q},{ }^{3} J_{\mathrm{HH}}=\right.$ $7.0 \mathrm{~Hz}, \mathrm{CH}_{2}$, of ether), $6.81(1 \mathrm{H}, \mathrm{s}, \mathrm{H} 5) ;{ }^{13} \mathrm{C} \mathrm{NMR}\left(125.75 \mathrm{MHz}, \mathrm{CDCl}_{3}\right) ; \delta=11.7,14.19$ 15.0, (3 $\left.\mathrm{CH}_{3}\right), 20.1,20.5$, (2 Me), 60.8, $69.5\left(2 \mathrm{OCH}_{2}\right), 90.7$ (C $\mathrm{C}$ Siro), 107.9 (C3a), 108.2 (C7), 122.2 (C6), 128.0 (C5), 129.1 (C4), 139.2 (C7a), 150.9 (C4'), 151.2 (C3'), 155.6, 161.6, 162.6 (3 C=O); MS, m/z (\%): $344\left(\mathrm{M}^{+}-1-\mathrm{Me}, 22\right), 315\left(\mathrm{M}^{+}-\mathrm{OCH}_{2} \mathrm{CH}_{3}, 16\right), 299$ $\left(\mathrm{M}^{+}-\mathrm{OCH}_{2} \mathrm{CH}_{3}-\mathrm{CH}_{3}, 4\right), 270\left(\mathrm{M}^{+}+1-\mathrm{OCH}_{2} \mathrm{CH}_{3}-\mathrm{CH}_{2} \mathrm{CH}_{3}-\mathrm{CH}_{3}, 100\right), 243 \quad\left(\mathrm{M}^{+}+1-\right.$ $\left.\mathrm{CO}_{2} \mathrm{CH}_{2} \mathrm{CH}_{3}-3 \mathrm{Me}, 12\right), 215\left(\mathrm{M}^{+}+1-\mathrm{CO}_{2} \mathrm{CH}_{2} \mathrm{CH}_{3}-3 \mathrm{Me}-\mathrm{CO}, 11\right), 91\left(\mathrm{C}_{7} \mathrm{H}_{8}{ }^{+}, 18.5\right) ; \mathrm{IR}(\mathrm{KBr})$ $\left(v_{\max }, \mathrm{cm}^{-1}\right): 3010\left(\mathrm{C}-\mathrm{H}_{\mathrm{St}}\right), 1752,1740,1705(3 \mathrm{C}=\mathrm{O}), 1585,1485(\mathrm{C}=\mathrm{C})($ arom $)$; Anal. calc. for $\mathrm{C}_{19} \mathrm{H}_{20} \mathrm{O}_{7}$ (360.36): C 63.33, H 5.59\% found: C 64.5, H 5.6\%. 


\section{Acknowledgements}

We gratefully acknowledge financial support from the Research Council of the University of Birjand

\section{References}

1. Mathews, C. J.; Taylor, J.; Tyte, M. J.; Worthington, P. A. Synlett 2005, 538.

2. Vale-Silva, L. A.; Buchta, V.; Vokurkova, D.; Pour, M. Bioorg. Med. Chem. Lett. 2006, 16, 2492.

3. Janecki, T.; Błaszczyk, E.; Studzian, K.; Janecka, A.; Krajewska, U.; Rozualski, M. J. Med. Chem. 2005, 48, 3516.

4. Pour, M.; Spulak, M.; Balsanek, V.; Kunes, J.; Kubanva, P.; Buchta, V. Biorg. Med. Chem. 2003, 11, 2843.

5. Gein, V. L.; Gein, L. F.; Sheptukha, M. A.; Voronina, E. V. Pharm. Chem. J. 2005, 39, 537.

6. Chatani, N.; Amako, K.; Tobisu, M.; Asaumi, T.; Fukumoto, Y.; Murai, S. J. Org. Chem. 2003, 68, 1591.

7. Domling, A.; Ugi, I. Angew. Chem. Int. Ed. 2000, 39, 3168.

8. Winterfeldt, E. Angew. Chem. Int. Ed. 1967, 6, 423.

9. Zhu, J.; Bienayme, H. Eds., Multicomponent Reactions, Wiley-VCH Verlag: Weinheim, 2005.

10. Ramazani, A.; Kazemizadeh, A. R.; Ahmadi, E.; Noshiranzadeh, N.; Souldozi,A. Curr. Org. Chem. 2008, 12, 59.

11. Ohno, H.; Ohta, Y.; Oishi, S.; Fujii, N. Angew. Chem. Int. Ed. 2007, 46, 2295.

12. Bonne, D.; Dekhane, M.; Zhu, J. P. Angew. Chem. Int. Ed. 2007, 46, 2485.

13. Yoshida, H.; Fukushima, H.; Ohshita, J.; Kunai, A. J. Am. Chem. Soc. 2006, 128, 11040.

14. Pache, S.; Lautens, M. Org. Lett. 2003, 5, 4827.

15. Siamaki, A. R.; Arndtsen, B. A. J. Am. Chem. Soc. 2006, 128, 6050.

16. Nair, V.; Nair, J. S.; Vinod, A. U.; Rath, N. P. J. Chem. Soc., Perkin Trans. 1 1997, 3129.

17. Nair, V.; Nair, J. S.; Vinod, A. U. Synthesis 2000, 1713.

18. Nair, V.; Deepthi, A.; Beneesh, P. B.; Eringathodi, S. Synthesis 2006, 1443.

19. Esmaeili, A. A.; Bodaghi, A. Tetrahedron 2003, 59, 1169.

20. Esmaeili, A. A.; Zendegani, H. Tetrahedron 2005, 61, 4031.

21. Johnson, A. W.; Tebby, J. C. J. Chem. Soc. 1961, 2126.

22. Tebby, J. C.; Wilson, I. F.; Grifiths, D. V. J. Chem. Soc., Perkin Trans. 1 1979, 2133. 